\title{
Native blood speckle vs ultrasound contrast agent for particle image velocimetry with ultrafast ultrasound - in vitro experiments
}

Jason Voorneveld ${ }^{1}$, Pieter Kruizinga ${ }^{1,4}$, Hendrik J. Vos $^{1}$, Frank J.H. Gijsen ${ }^{1}$, Erik Groot Jebbink ${ }^{2,3}$, Antonius F.W. van der Steen ${ }^{1,4,5,6}$, Nico de Jong ${ }^{1,4}$, Johan G. Bosch ${ }^{1}$

${ }^{1}$ Department of Biomedical Engineering, Thorax Center, Erasmus MC, Rotterdam, Netherlands,

${ }^{2}$ Department of Surgery, Rijnstate Hospital, Arnhem, Netherlands,

${ }^{3}$ MIRA Institute for Biomedical Technology and Technical Medicine, University of Twente, Enschede, Netherlands,

${ }^{4}$ Laboratory of Acoustical Wavefield Imaging, Faculty of Applied Sciences, Delft University of Technology, Delft, Netherlands

${ }^{5}$ Interuniversity Cardiology Institute, Utrecht, The Netherlands

${ }^{6}$ Shenzhen Institutes of Advanced Technology, Chinese Academy of Sciences, Shenzhen, China

\begin{abstract}
Ultrafast contrast enhanced ultrasound, combined with echo particle image velocimetry (ePIV), can provide accurate, multidimensional hemodynamic flow field measurement. However, the use of ultrasound contrast agent (UCA) still prevents this method from becoming a truly versatile and non-invasive diagnostic tool. In this study, we investigate the use of native blood instead of UCA backscatter for ePIV measurements and compare their accuracy in vitro. Additionally, the effect of measurement depth is experimentally assessed.
\end{abstract}

Blood mimicking fluid (BMF) was pumped through a $10 \mathrm{~mm}$ diameter tube producing parabolic flow profiles, adding UCA in the case of contrast imaging. Plane wave imaging at 5000 framesper-second was performed with a Verasonics Vantage system and a linear array. The tube was imaged at three different depths: 25, 50 and $100 \mathrm{~mm}$. Singular value decomposition (SVD) was assessed for clutter suppression against mean background subtraction. PIVlab was used as a PIV implementation.

With SVD, BMF provided almost equal ePIV accuracy as UCA, except at $100 \mathrm{~mm}$ depth where UCA provided better accuracy. Use of clutter suppression greatly improved ePIV results, but minimal differences in ePIV accuracy were noted between mean and SVD filtered groups (BMF or UCA). Accuracy decreased with increasing depth, likely due to reduced elevation resolution, resulting in out-of-plane smoothing of velocity gradients.

Keywords-Ultrafast Imaging; Ultrasound Contrast Agent; Particle Imaging Velocimetry; Speckle Tracking; Clutter Suppression

\section{INTRODUCTION}

Visualization and quantification of blood velocity perpendicular to the ultrasound (US) beam direction remains a challenge in clinical imaging. This is especially true when imaging deep structures such as in the heart or abdominal aorta. In this case, vector imaging techniques such as Vector Doppler imaging suffer reduced accuracy due to limited probe aperture.

This research is supported by ZonMw within the Innovative Medical Devices Initiative (IMDI) program (project Heart Failure and 4D Flow).
A different approach for estimating velocity is by echoparticle imaging velocimetry (ePIV), which instead operates on successive, beamformed frames, in a patch-by-patch basis, to determine velocity fields. While ePIV was first demonstrated by Kim et al in 2004 [1], its success has been limited due to the relatively low frame-rates attainable in conventional ultrasound scanners $(<100 \mathrm{fps})$. Low frame rate results in decorrelation of high velocity flows due to the relatively large particle displacement between frames. The recent advances in ultrafast ultrasound imaging have been shown to offer a solution to this limitation of ePIV [2]. However, the use of ultrasound contrast agent (UCA) still prevents ePIV from becoming a truly versatile and non-invasive diagnostic tool.

In this study, we compare the use of UCA and speckle from blood mimicking fluid (BMF, as a proxy for native blood speckle) as signal sources for ePIV, after using singular value decomposition (SVD, [3]) as a clutter filter. Additionally, we perform the comparison at different depths, in an effort to investigate the relationship between ePIV accuracy and depth.

\section{Methods}

\section{A. In Vitro Phantom}

A custom designed in vitro flow phantom was used to pump blood mimicking fluid (BMF) through a 10mm diameter tube. The tube was immersed in water to allow for ultrasonic imaging at 3 different depths from the transducer lens $(25,50$ and $10 \mathrm{~mm}$ ). Calibrated flow rates, corresponding to parabolic velocity profiles, were measured with an inline ultrasonic flow meter (UF Ultrasonics Flow Meter, Cynergy ${ }^{3}$ Components, UK, 3\% accuracy). Three physiologically relevant peak velocities $(0.25,0.5$ and $0.75 \mathrm{~m} / \mathrm{s})$ were studied.

BMF was prepared according the recipe of Ramnarine et al. with $5 \mu \mathrm{m}$ Orgasol particles, providing equivalent backscatter to human blood [4]. A diluted, commercial UCA (SonoVue, $50 \mu 1 / 1$ concentration) was added to the BMF in the case of contrast imaging. 


\section{B. Ultrafast Ultrasound Acquisition and Beamforming}

Raw channel data were acquired with a Verasonics Vantage 256 system (Verasonics Inc., USA) using an ATL L7-4 transducer $(5 \mathrm{MHz}, 25 \mathrm{~mm}$ elevation focus, $298 \mu \mathrm{m}$ pitch, 128 elements). A plane wave acquisition protocol captured 300 frames at the pulse repetition frequency (PRF) of $5000 \mathrm{~Hz}$. Three different clutter suppression techniques were compared by applying each filter on the pre-beamformed channel data: a) no filtering, b) mean subtraction (along slow time) and c) hard threshold SVD rank reduction (keeping ranks 5 to 270).

Beamforming was performed offline, using a Fast Fourier Transform (FFT) method [5]. Beamformed RF data was sincinterpolated in the axial direction to obtain equal pixel spacing in axial and lateral direction. Finally, the envelope was detected and log compressed forming B-mode image sets.

\section{C. $e P I V$}

PIVlab (V1.41,[6]) was used as a Particle Imaging Velocimetry implementation in Matlab (R2015a, The MathWorks Inc., Natick, MA). PIVlab utilizes an iterative approach to ePIV, deforming the target kernel after each iteration according to the previous iteration's displacement estimates, theoretically converging towards true autocorrelation. In this study three iterations, with square kernel sizes of $2.3 \times 2.3,2.3 \times 2.3$ and $1.15 \times 1.15 \mathrm{~mm}^{2}$ for each successive iteration, were used, using bicubic interpolation for image deformation of the target image after each iteration. Post processing was limited to discarding the velocity estimates on the borders of the region of interest, no outlier elimination or smoothing was applied. ePIV was performed on every $5^{\text {th }}$ frame (effective frame rate of 1000).

\section{Analysis \& Statistics}

A ground truth velocity profile was calculated using the Hagen-Poiseuille equation for pipes of circular cross section, $v_{r}=\left(Q / \pi R^{2}\right)\left\lceil 1-\frac{r^{2}}{R^{2}}\right\rceil ;$ where $v_{r}$ is the velocity at radius $r$ within the total pipe radius $R(r \leq R)$ and $Q$ is the flow rate measured by the flow meter. This study compared the signal obtained from UCA and BMF with 3 different filters (none, mean subtraction and SVD rank reduction) at 3 depths $(25,50$ and $100 \mathrm{~mm}$ ) with 3 repeated experiments per group.

Bias error was defined as the Mean of the Absolute Difference (MAD) between the ePIV derived profile and the ground truth profile. The MAD was normalized to the peak ground truth velocity allowing for comparison over different flow rates: $\epsilon=\sum_{i=1}^{N}\left|v_{P I V(i)}-v_{G T(i)}\right| /\left(\mathrm{N} \times \max \left(v_{G T}\right)\right)$; where $\epsilon, v_{i}$ and $N$ are the error, velocity (ePIV or ground truth) at point $i$, and $\mathrm{N}$ is the total number of ePIV sampled points (along the radius of the tube), respectively. Vectors were averaged over ten consecutive frames before calculating the MAD.

Comparison between groups were performed by means of 2-way ANOVA followed by multiple comparisons using an uncorrected Fisher's LSD in GraphPad Prism (v7.01, GraphPad Software, USA).

\section{RESULTS}

SVD filtering suppressed the tube wall and its reverberation artefacts better than mean amplitude subtraction, as depicted in Fig. 1, where mean subtraction was not able to completely suppress the tube wall. After SVD clutter suppression, cineloops of BMF and UCA were visually similar (40dB
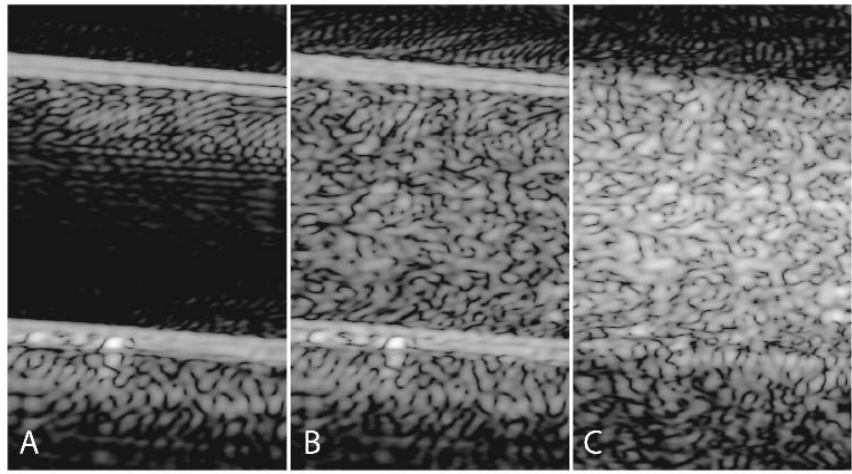

Fig. 1. Effects of different clutter filters on BMF: A) no filter, B) mean subtraction and C) SVD. Mean amplitude subtraction fails to suppress the tiny vibrations in the tube wall (likely caused by the pumps). SVD successfully suppresses clutter. Dynamic range of $40 \mathrm{~dB}$.
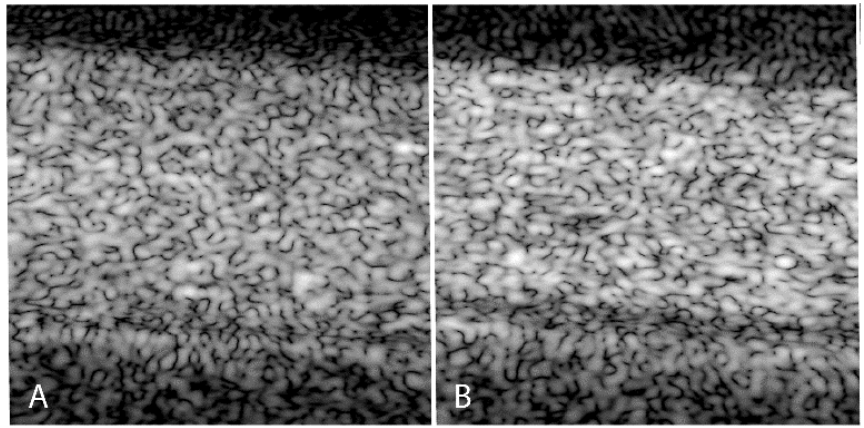

Fig. 2. B-mode image sections of tube after SVD filtering for A) BMF and B) UCA at a dynamic range of $40 \mathrm{~dB}$.

dynamic range, Fig. 2).

The profile estimated by ePIV was similar to the theoretical profile calculated from the measured flow rate. This is depicted in Fig. 3. with ePIV vectors and theoretical ground truth overlaid on a single B-mode frame.

The effect of clutter filtration (mean over the depths and velocities sampled in this study) is summarized in Fig. 4. Without clutter filtration UCA performed far better than BMF ( $27 \pm 16 \%$ vs $67 \pm 5 \%$ bias error, respectively). Utilizing SVD however, there was minimal difference in result, only at 100 mm depth did UCA provide improved ePIV accuracy over BMF $(13 \pm 6 \%$ vs $19 \pm 6 \%$, $\mathrm{p}=0.005)$. No significant differences were observed between mean amplitude subtraction and SVD filtered ePIV results (BMF and UCA). 


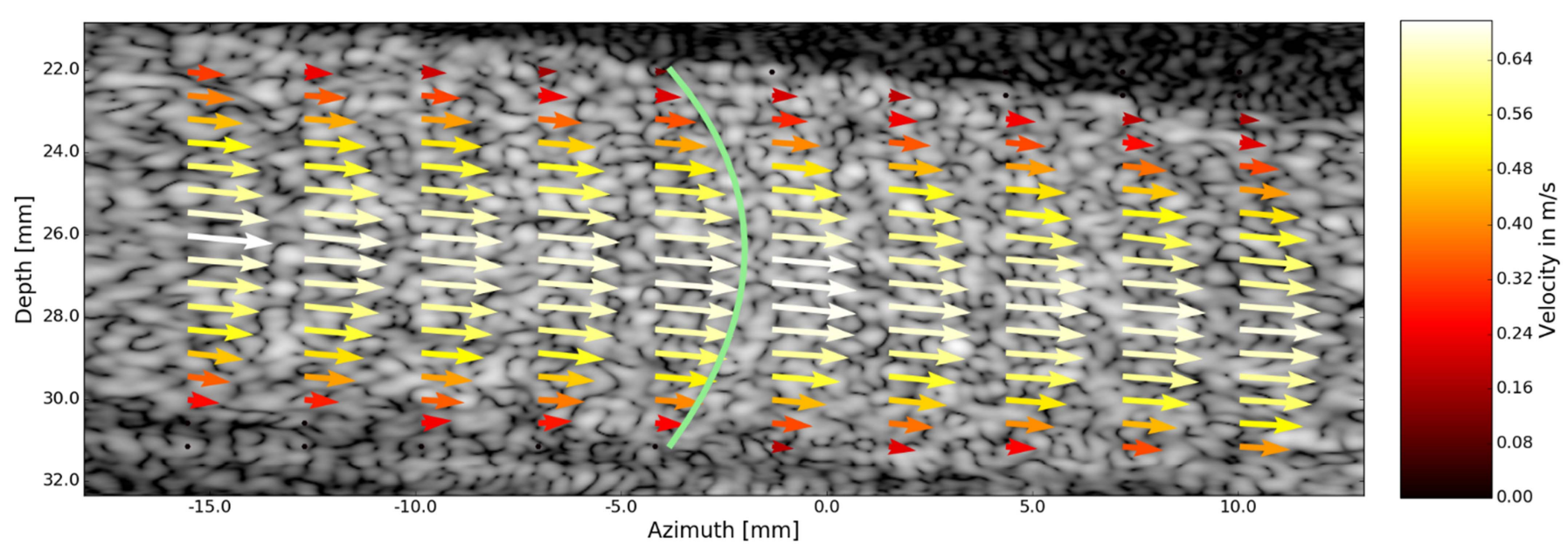

Fig. 3. Example of ePIV result (arrows) at $25 \mathrm{~mm}$ depth. Green curve depicts theoretical velocity profile calculated from the measured flow meter data

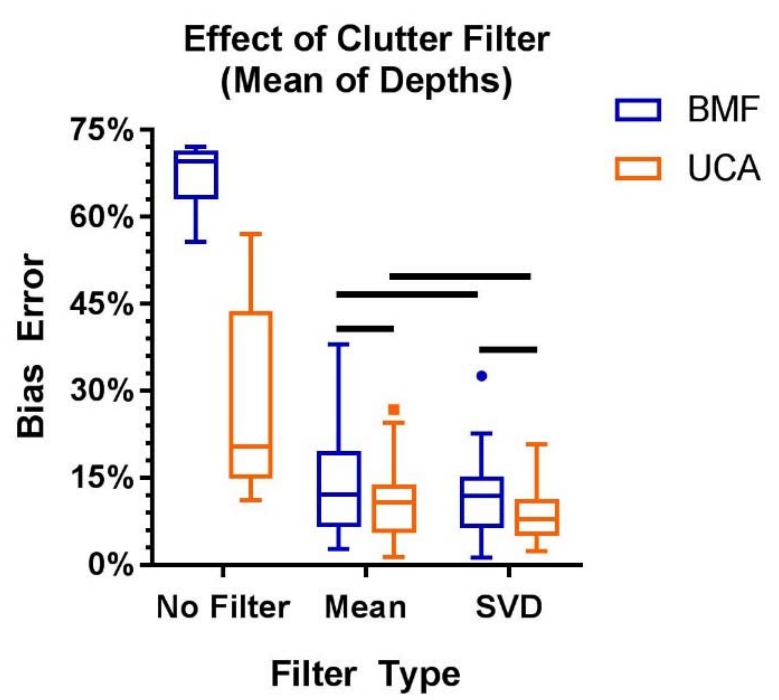

Fig. 4. Effect of clutter filter on the resultant ePIV bias. Using a clutter filter significantly improves accuracy for both BMF and UCA. $n=9$. *solid lines denote $\mathrm{p}>0.05$ (no significance)

With respect to Fig. 5., depth was a significant factor in ePIV accuracy $(\mathrm{p}<0.0001)$, with mean bias increasing with depth, even after clutter suppression using SVD filtering. Error values (using SVD) at 25, 50 and $100 \mathrm{~mm}$ were $4 \%, 12 \%$ and $19 \%$ for BMF and $5 \%, 9 \%$ and $13 \%$ for UCA, respectively (Fig. 5).

\section{DISCUSSION}

\section{A. Findings}

With clutter suppression, BMF provided almost equal ePIV accuracy as UCA, except at $100 \mathrm{~mm}$ depth, where BMF's signal-to-noise ratio (SNR) was lower. SVD rank reduction was more effective at clutter filtering than mean subtraction,

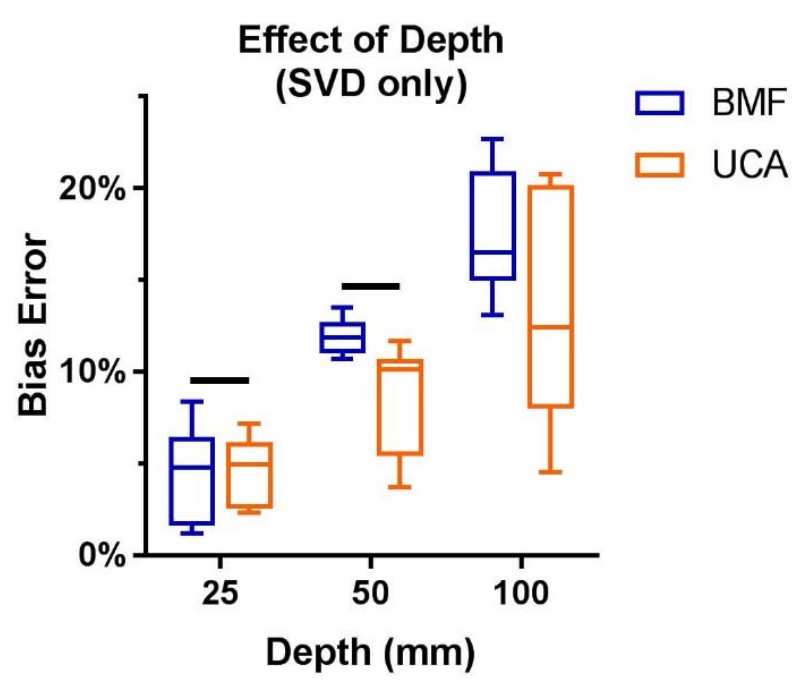

Fig. 5. Effect of increasing depth on ePIV bias error. For both BMF and UCA increasing depth results in increased bias error. The effect is stronger for BMF resulting in poorer performance than UCA at $100 \mathrm{~mm}$ depth. $\mathrm{n}=9$. *solid lines denote $\mathrm{p}>0.05$ (no significance)

which was not able to completely suppress the signal from the tube wall due to sub-wavelength motion in the wall, induced by pump vibrations. However, the superiority of SVD in clutter suppression did not transfer to improved ePIV results over the mean method. This was most likely due to the region of interest that was used for calculating the ePIV, which was limited to the lumen of the tube.

With the aid of clutter suppression, ePIV accurately estimated the velocity profile within the tube. Similar accuracy to Leow et al. (2015) was obtained when imaging at $25 \mathrm{~mm}$ depth [2]. However, it should be noted that in the current study no contrast specific acquisition scheme was used. Contrast specific acquisition schemes such as pulse inversion or 
amplitude modulation would isolate blood signal and suppress tissue signal, at the cost of reduction of the frame rates by a factor 2-3. Yet, SVD filtering already isolates blood signal, thus negating the need of a contrast mode. Alternatively, compounding techniques [7] have a similar cost of frame rate reduction, but may improve image resolution and SNR, which would likely further improve ePIV performance.

The persistent underestimation of velocity with increasing depth was suspected to be due to the increased beam-width in the elevational direction as depth increases. In this case, with the circular cross section of the tube, causing velocity averaging of the parabolic profile over the beam width. This can readily be verified by repeating the experiments with a transducer with a deeper elevation focus.

\section{B. Limitations}

The use of water as a propagation medium for ultrasound resulted in minimal attenuation and phase aberration in the ultrasound signal. It is possible that the attenuation in vivo will result in too low SNR from the native blood speckle for even the SVD algorithm to enhance, especially at great depth. Also, there is far more considerable tissue motion in vivo than the sub-wavelength vibrations experienced in the current study. It remains to be seen how well SVD separates clutter, blood and noise signal in large vessels in vivo. Also, the current implementation of SVD utilized a manual selection of the eigenvalues threshold, by which tissue clutter, flow signal and noise are separated. An automatically calculated threshold would be an improvement and will be explored further. Finally, the current study did not report velocities greater than $0.75 \mathrm{~m} / \mathrm{s}$, this was a limitation of the flow phantom when used with BMF and not ePIV. When exceeding $0.75 \mathrm{~m} / \mathrm{s}$ the system sucked airbubbles into the flow medium which made comparison between UCA and BMF impossible.

\section{CONCLUSION}

The use of ultrafast ultrasound imaging in conjunction with ePIV allowed for accurate flow velocity estimation in vitro. Significant improvements in ePIV accuracy, through the addition of UCA, only occurred at $100 \mathrm{~mm}$ depth.

\section{ACKNOWLEDGMENT}

We acknowledge G. Springeling and A. Brouwer for their assistance in constructing the phantom.

\section{REFERENCES}

[1] H. B. Kim, J. R. Hertzberg, and R. Shandas, "Development and validation of echo PIV," Exp. Fluids, vol. 36, no. 3, pp. 455-462, 2004.

[2] C. H. Leow, E. Bazigou, R. J. Eckersley, A. C. H. Yu, P. D. Weinberg, and M.-X. Tang, "Flow Velocity Mapping Using Contrast Enhanced High-Frame-Rate Plane Wave Ultrasound and Image Tracking: Methods and Initial in Vitro and in Vivo Evaluation," Ultrasound Med. Biol., vol. 012, pp. 1-13, 2015.

[3] C. Demene, T. Deffieux, M. Pernot, B.-F. Osmanski, V. Biran, J.-L. Gennisson, L.-A. Sieu, A. Bergel, S.
Franqui, J.-M. Correas, I. Cohen, O. Baud, and M. Tanter, "Spatiotemporal Clutter Filtering of Ultrafast Ultrasound Data Highly Increases Doppler and fUltrasound Sensitivity," IEEE Trans. Med. Imaging, vol. 34, no. 11, pp. 2271-2285, Nov. 2015.

[4] K. V. Ramnarine, D. K. Nassiri, P. R. Hoskins, and J. Lubbers, "Validation of a new blood-mimicking fluid for use in Doppler flow test objects," Ultrasound Med. Biol., vol. 24, no. 3, pp. 451-459, 1998.

[5] J. Y. Lu, "2D and 3D High Frame Rate Imaging with Limited Diffraction Beams," IEEE Trans. Ultrason. Ferroelectr. Freq. Control, vol. 44, no. 4, pp. 839856, 1997.

[6] W. Thielicke and E. J. Stamhuis, "PIVlab - Towards User-friendly, Affordable and Accurate Digital Particle Image Velocimetry in MATLAB," J. Open Res. Softw., vol. 2, 2014.

[7] M. Tanter and M. Fink, "Ultrafast imaging in biomedical ultrasound," IEEE Trans. Ultrason. Ferroelectr. Freq. Control, vol. 61, no. 1, pp. 102119, 2014. 